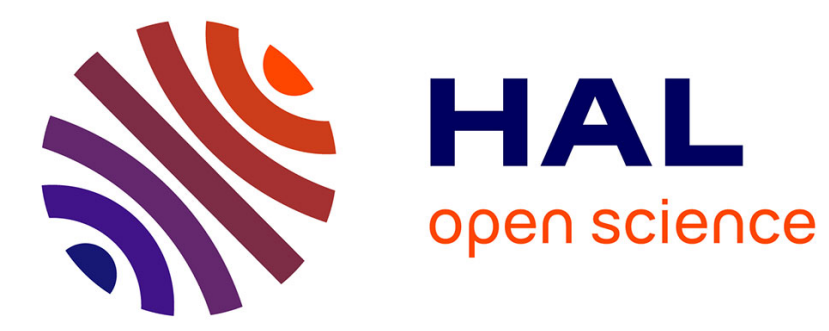

\title{
Pulse doublets generated by a frequency-shifting loop containing an electro-optic amplitude modulator
}

Hongzhi Yang, Marc Vallet, Haiyang Zhang, Changming Zhao, Marc Brunel

\section{To cite this version:}

Hongzhi Yang, Marc Vallet, Haiyang Zhang, Changming Zhao, Marc Brunel. Pulse doublets generated by a frequency-shifting loop containing an electro-optic amplitude modulator. Optics Express, 2019, 27 (13), pp.18766-18775. 10.1364/OE.27.018766 . hal-02163678

\section{HAL Id: hal-02163678}

\section{https://hal-univ-rennes1.archives-ouvertes.fr/hal-02163678}

Submitted on 24 Jun 2019

HAL is a multi-disciplinary open access archive for the deposit and dissemination of scientific research documents, whether they are published or not. The documents may come from teaching and research institutions in France or abroad, or from public or private research centers.
L'archive ouverte pluridisciplinaire HAL, est destinée au dépôt et à la diffusion de documents scientifiques de niveau recherche, publiés ou non, émanant des établissements d'enseignement et de recherche français ou étrangers, des laboratoires publics ou privés. 


\title{
Pulse doublets generated by a frequency- shifting loop containing an electro-optic amplitude modulator
}

\author{
Hongzhi YANG, ${ }^{1,2}$ Marc VAllet ${ }^{2}$ HaiYAng Zhang, ${ }^{1}$ Changming ZhaO, ${ }^{1}$ \\ AND MARC BRUNEL ${ }^{2, *}$ \\ ${ }^{I}$ School of Optics and Photonics, Beijing Institute of Technology, Beijing, China \\ ${ }^{2}$ Univ Rennes, CNRS, Institut FOTON - UMR 6082, 35000 Rennes, France \\ *marc.brunel@univ-rennes1.fr
}

\begin{abstract}
We investigate theoretically and experimentally an all-fibered frequency-shifting loop which includes an electro-optic amplitude modulator (EOM) and an optical amplifier, and is seeded by a continuous-wave laser. At variance with frequency-shifted feedback lasers, or Talbot lasers, that contain an acousto-optic frequency shifter, the EOM creates at each round-trip two side-bands that recirculate inside the loop. Benefiting from the high modulation frequency of the EOM, a wide optical frequency comb up to $40 \mathrm{GHz}$ is generated. We demonstrate an original double-pulse regime when the loop length is a multiple of the RF modulation wavelength applied to the modulator. The inter-pulse interval is governed by both the bias voltage and modulation depth of the EOM. Besides, some typical waveforms such as saw-tooth and rectangle are experimentally obtained by properly setting operating frequency, bias voltage and the RF power. The system is modeled by a linear interference model that takes the amplitude modulation function and loop delay into account. The model explains the formation of pulse doublets and reproduces well all the experimental waveforms. Furthermore, the un-seeded loop driven above threshold also generates mode-locked picosecond pulse doublets with a continuously adjustable delay up to the modulation period.
\end{abstract}

(C) 2019 Optical Society of America under the terms of the OSA Open Access Publishing Agreement

\section{Introduction}

Microwave photonics is an innovative multi- and interdisciplinary field that investigates the interaction between microwave and optical signals including microwave signal generation and processing [1-3], microwave-photonic systems [4,5], and broadband optical links for high-speed interconnects [6]. In particular, the generation of high repetition-rate optical pulses plays an important role in high-speed optical fiber and microwave photonics systems $[7,8]$. In this respect, frequency-shifting loops (FSL), that are loop resonators containing both an amplifier and an acousto-optic frequency-shifter (AOFS), have been demonstrated to be promising solutions to generate Fourier-transform-limited pulses with tunable and ultrahigh repetition rates [9-11]. When the frequency shift is tuned to a fraction of the cavity freespectral range, periodic pulse trains can be generated from a continuous-wave seed laser, leading to a so-called "Talbot laser" due to the complete analogy with the spatial Talbot effect [12]. Similar all-fiber set-ups have also been extended to applications such as high data-rates in radio-over-fiber communications [13,14], real-time Fourier transformation of optical signals [15] and have also recently been shown to produce arbitrary waveform generation [16].

Frequency-shifted loops usually rely on the use of an acousto-optic frequency-shifter. While it features high frequency conversion efficiency in the sub-100 MHz range, AOFS have limited efficiency in the GHz range, and offer limited tunability. In this respect, EOM offer much higher modulation frequency and bandwidth. Besides, EOM are compact and easy to integrate with other fibered devices. Few studies investigated single-sideband EOM 
operation in FSLs: a multi-carrier source was built with high power flatness and stability [14], and recently $\mathrm{GHz}$ repetition rates were demonstrated in the Talbot configuration [17]. In this article, we investigate an all-fibered frequency-shifted feedback loop when a widely tunable common, dual side-band, electro-optic amplitude modulator (EOM) is employed. Instead of the single side-band AO frequency-shift or SSB-EOM, the loop will produce at each roundtrip two side-bands with opposite frequency-shifts. The carrier will also circulate together with the multiple frequency-shifted sidebands. This raises questions about the ability to generate a pulse train from a continuous-wave seed, the so-called continuous-to-pulse conversion regime [11,12], or to generate arbitrary waveforms $[16,18]$. Furthermore, an analytical model has to be derived in order to take into account the specific transfer function of the EOM.

We first present the method and the corresponding model in Section 2. In particular, we focus on the integer Talbot condition where predictions can be derived from simple algebra. Section 3 presents the experimental results obtained with standard components at $1.55 \mu \mathrm{m}$ wavelength, looking at the influences of the EOM parameters on the FSL properties. Pulse train generation and specific waveforms are investigated by precisely controlling the modulating frequency, RF power and the bias voltage applied to the EOM. Then Section 4 is devoted to the extension of the method to the un-seeded, mode-locked laser operation, and the comparison with the results of Section 3. Finally, conclusions and perspectives are included in Section 5.

\section{Method}

\subsection{Set-up principle}

We consider the fiber loop depicted in Fig. 1. It contains an electro-optic amplitude modulator $(\mathrm{EOM})$ that induces a dual-sideband frequency shift per round-trip $f_{m}$, and an erbium-doped fiber amplifier (EDFA) providing gain that partially compensates for the loop losses and enhances the number of relevant round-trips inside the loop, as in AOFS-based loops. Besides, an optical filter permits to limit the output bandwidth and efficiently reduces parasitic loop oscillations, while a polarization controller also stabilizes the loop operation. The round-trip time is $\tau=n L / c$, where $n$ is the group index of the loop fiber. This leads to a fundamental loop frequency $f_{c}=1 / \tau$. A $2 \times 2$ optical coupler enables to seed the loop and to extract a fraction of the circulating laser power. The EOM is driven by a radiofrequency synthesizer (SYN) and a bias voltage (DC). We assume that the loop is below the laser threshold, i.e., the gain does not compensate for the losses. The setup is similar to the one of Refs [11-13] but the frequency-shift is provided by a common EOM instead of an AOFS.

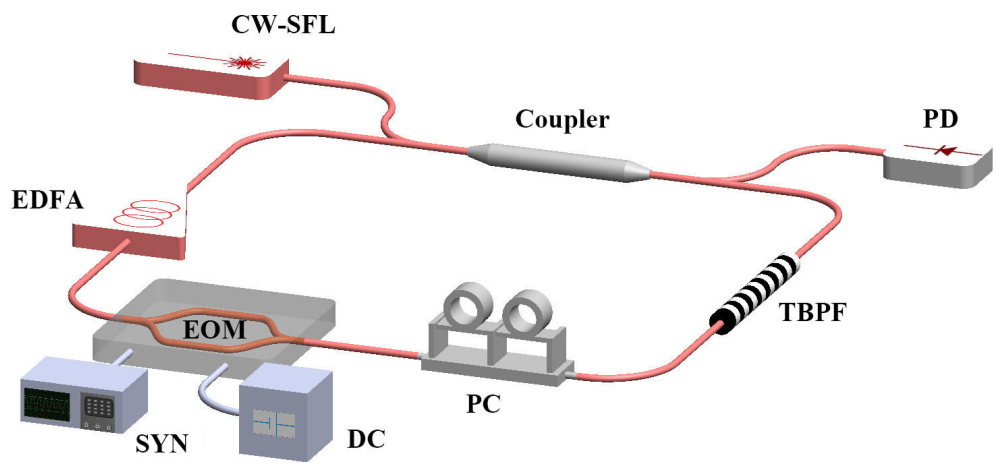

Fig. 1. Sketch of the dual side-band FS loop. CW-SFL: continuous-wave single-frequency laser; PD: photodiode; TBPF: tunable bandpass filter; PC: polarization controller; EOM: Mach-Zehnder intensity modulator driven at frequency $f_{m}(\mathrm{SYN})$ and bias voltage $V_{b}(\mathrm{DC})$; EDFA: erbium-doped Optical Fiber Amplifier. 


\subsection{Model}

In order to predict the output waveform, we derive a time-delayed interference model. Given the transmission matrix of the coupler $\left[t_{\mathrm{ij}}\right]$ and $E_{\text {in }}$ the input electric field of the coupler, the output field $E_{\text {out }}$ can be written as follows:

$$
\left[\begin{array}{c}
E_{\text {out } 1} \\
E_{\text {out } 2}
\end{array}\right]=\left[\begin{array}{ll}
t_{11} & t_{12} \\
t_{21} & t_{22}
\end{array}\right]\left[\begin{array}{c}
E_{\text {in1 }} \\
E_{\text {in } 2}
\end{array}\right] \text {. }
$$

In the case of a lossless coupler, the $t_{i j}$ verify the condition $t_{11} t_{22}-t_{12} t_{21}=1$ [19]. At round-trip $p$, the real transfer function of the EOM can be modeled as $\mathrm{Y}^{(p)}(t)=\sin \left[\Gamma+\Gamma_{m} \sin \left(2 \pi f_{m}(t-\right.\right.$ $p \tau)$ )], where $\Gamma_{m}$ is the modulating depth that depends on the RF power $\mathrm{P}_{\mathrm{dc}} . \Gamma$ is the static phase retardance of the EOM that can be controlled by the applied bias voltage $V_{b}$. If $\eta$ and $G$ are the intensity loss of the loop and the intensity gain parameters, respectively, then we write $\gamma=\sqrt{\eta G}$ the overall amplitude transmission. To find out the field circulating inside the loop, we calculate the electric field at output port 2. From Eq. (1), this field writes:

$$
E_{\text {out } 2}(t)=t_{21} E_{\text {in } 1}+t_{22} E_{\text {in } 2},
$$

from which one gets:

$$
E_{\text {out } 2}(t)=t_{21} E_{\text {in1 }}(t)+t_{22} \gamma E_{\text {out } 2}(t-\tau) \Upsilon^{(1)}(t) .
$$

This formulation can be expanded using

$$
E_{\text {out } 2}(t-\tau)=t_{21} E_{\text {in1 }}(t-\tau)+t_{22} \gamma E_{\text {out } 2}(t-2 \tau) \Upsilon^{(2)}(t) .
$$

Inserting Eq. (4) into the right-hand side of Eq. (3), the equation can be expanded to $\mathrm{N}$ roundtrips in the loop:

$$
E_{\text {out } 2}(t)=t_{21} E_{\text {in } 1}(t)+\sum_{p=1}^{N} t_{21} t_{22}^{p} \gamma^{p} \prod_{l=1}^{p} \Upsilon^{(l)}(t) E_{\text {in1 }}(t-p \tau) .
$$

The experimentally accessible and useful signal is at the output port 1 . If the input field at port 1 is a single-frequency continuous-wave with power $P_{i n}$, then

$$
E_{\text {out } 1}(t)=t_{11} \sqrt{P_{\text {in }}}+t_{21} t_{12} \sum_{p=1}^{N} t_{22}^{p-1} \gamma^{p} \prod_{l=1}^{p} \Upsilon^{(l)}(t) \sqrt{P_{\text {in }}},
$$

from which the power $P_{\text {out }}(t)$ can be derived:

$$
P_{\text {out }}(t)=\left|t_{11}+t_{21} t_{12} \sum_{p=1}^{N} t_{22}^{p-1} \gamma^{p} \prod_{l=1}^{p} \Upsilon^{(l)}(t)\right|^{2} P_{\text {in }} .
$$

In the following, we use Eq. (7) to calculate the output waveform. Note that contrary to the theoretical model developed for AOFS loops [11,12], here no simple analytical formula can be deduced. However, under the integer Talbot conditions $f_{m}=n f_{c}$, where $n$ is an integer, we find $\sin \left[2 \pi f_{m}(t-p \tau)\right]=\sin \left(2 \pi f_{m} t\right)$. Then, in the limit $N \rightarrow+\infty$, the sum of the geometric series in Eq. (7) can be simplified, leading to

$$
P_{\text {out }}(t)=\left|t_{11}+\frac{t_{21} t_{12} \gamma \sin \theta(t)}{1-t_{22} \gamma \sin \theta(t)}\right|^{2} P_{\text {in }},
$$


where we introduced $\theta(t)=\Gamma+\Gamma_{m} \sin \left(2 \pi f_{m} t\right)$. Since $t_{22} \gamma \sin \theta(t)<1$ (assuming $t_{22}$ real positive), obviously $\sin \theta(t) /\left(1-t_{22} \gamma \sin \theta(t)\right)$ will be a sharp function peaked at $\theta(t)=\pi / 2$. As in the AOFSbased loops, pulses are found when the Talbot condition is met, i.e., when the modulation frequency is an integer number of times the loop frequency (or, equivalently, when the loop length is an integer number of times the beat length). It is interesting to note that Eq. (8) is independent of $n$, which means that the pulse shape is expected to be the same whatever the modulation frequency, and that the pulses will become shorter as frequency increases. According to Eq. (8), the important point, specific to our amplitude modulation case, is that two temporally separated solutions satisfy Eq. (8) in one period $1 / f_{\mathrm{m}}$. Indeed, we may predict that the response of the amplitude-modulated loop in the time domain delivers a periodic series of pulse doublets. The delay $\Delta t$ between the two pulses in one period $1 / f_{\mathrm{m}}$ is found to be

$$
\Delta t=\frac{1}{2 f_{m}}\left[1-\frac{2}{\pi} \sin ^{-1}\left(\frac{\pi / 2-\Gamma}{\Gamma_{m}}\right)\right] .
$$

This shows that $\Gamma$ and $\Gamma_{m}$ will have a strong influence on the delay. Sketches of the output time responses with different $\Gamma$ and $\Gamma_{m}$ are depicted in Figs. 2(a)-2(b). For example if $\Gamma=\pi / 2$ then $\Delta t=1 /\left(2 f_{m}\right)$, leading to a pulse repetition rate equal to twice the modulation frequency (red curve in Fig. 2(a)).
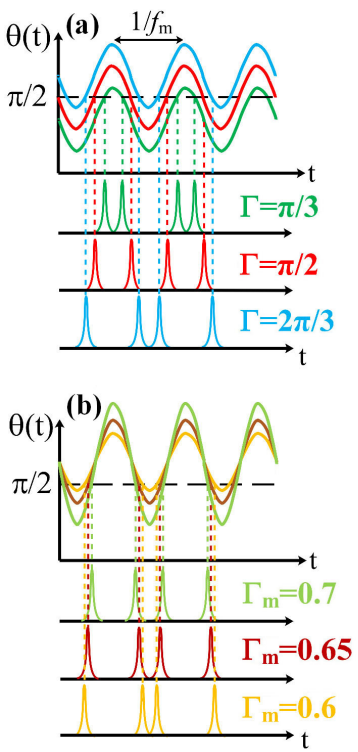

(c)

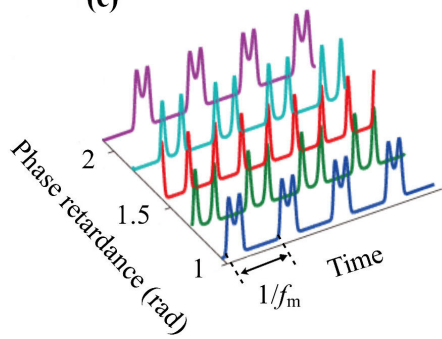

(d)

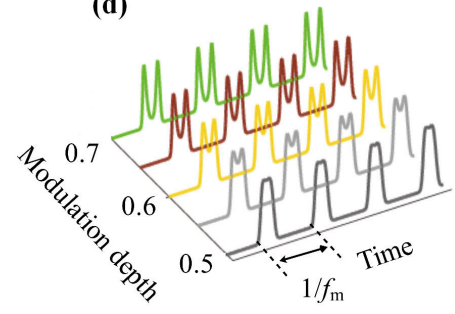

Fig. 2. Sketches of output signal vs time, with (a) different $\Gamma$ and (b) different $\Gamma_{\mathrm{m}}$. Simulation output power with (c) $\Gamma=\pi / 3$ (blue), $5 \pi / 12$ (green), $\pi / 2$ (red), $7 \pi / 12$ (light blue), and $2 \pi / 3$ (purple), and (d) $\Gamma_{\mathrm{m}}=0.5$ (black), 0.55 (grey), 0.6 (yellow), 0.65 (brown), and 0.7 (green).

To further illustrate the influence of $\Gamma$ and $\Gamma_{m}$ on the double-pulse operation, we perform simulations based on Eq. (7) with $\mathrm{N}=30$ round-trips. Figure 2(c) depicts the simulation results, with $\Gamma_{m}=0.7$ and $\gamma=0.9$ for example, showing that the delay between the two pulses increases with $\Gamma$. Then the influence of $\Gamma_{m}$ on the double-pulse is also simulated and depicted in Fig. 2(d) when $\Gamma=2 \pi / 3$. With the increase of $\Gamma_{m}$, the delay gradually approaches one half period. Note that $\Gamma_{m}$ also has an influence on the pulse width. Indeed, lower values of $\Gamma_{m}$ will directly reduce the width of the optical-carried RF comb in the optical frequency domain, hence leading to pulse widening. These predictions are tested experimentally in the following. 


\section{Pulse doublets experiments}

\subsection{Experimental parameters}

We experimentally investigate the time response of the dual side-band frequency-shifting loop as depicted in Fig. 1. Experiments are performed with a continuous-wave tunable singlefrequency semiconductor laser, which delivers $0.5 \mathrm{~mW}$ in the $\mathrm{C}$ band, with a linewidth of about $\Delta v=100 \mathrm{kHz}$. In order to avoid parasitic oscillations when the EDFA gain is raised, we use a $40 \mathrm{GHz}$-bandwidth $(0.3 \mathrm{~nm})$ optical filter inside the loop. Here, at variance with the FS loop of Refs [10-12] where the wavelength of the seed laser is at one edge of the optical filter (single-sideband frequency comb), we set the wavelength of our laser at the center of the optical filter around $1552 \mathrm{~nm}$ (dual-sideband frequency comb). From the measured values of the four intensity transmission coefficients, $\left|t_{i j}\right|^{2}$ the coupling matrix is set to $\left[t_{i j}\right]=\left[\begin{array}{cc}0.44 & 0.65 i \\ 0.43 i & 0.67\end{array}\right]$. The loop fundamental frequency is measured to be $f_{c}=6.737 \mathrm{MHz}$, corresponding to a loop delay time $\tau=0.148 \mu$ s and an optical length of $44.53 \mathrm{~m}$. Note that the coherence length $L_{\mathrm{c}}=c / \Delta v$ ( $c$ is the velocity of light in the medium) of the seed laser corresponds to more than 60 round-trips, exceeding the $\mathrm{N}=30$ round-trips used in the simulation. The polarization controller (PC) is utilized to stabilize the polarization state of the laser signal to make the modulation depth higher and waveform more stable. In order to minimize the output pulse width (or maximize the optical bandwidth), the polarization state of the laser and the pump current of the EDFA are adjusted jointly. The detection setup consists in a $40 \mathrm{GHz}$-bandwidth photodiode and a high-resolution optical spectrum analyzer. The signal in time domain is monitored with an $11 \mathrm{GHz}$-bandwidth and $40 \mathrm{Gs} / \mathrm{s}$ sampling-rate oscilloscope.

Finally, the EOM is a $10 \mathrm{GHz}$-bandwidth Mach-Zehnder modulator with a half-wave voltage of $4 \mathrm{~V}$. The FS loop output characteristics depend on RF frequency $f_{m}$, RF power $\mathrm{P}_{\mathrm{dc}}$, and bias voltage $V_{b}$.

\subsection{Dual-pulse regime}

In agreement with the theoretical results obtained with $f_{m}=n f_{c}$, we find that the time response of the dual side-band FS loop is a periodic train of the pulse doublets with a temporal period equal to the inverse of the RF frequency applied to the EOM, as shown in Fig. 3. Figures 3(a)-3(d) report the experimental results when $n=1\left(f_{m}=6.737 \mathrm{MHz}\right), 10(67.37 \mathrm{MHz}), 100$ $(673.7 \mathrm{MHz})$, and $500(3.369 \mathrm{GHz})$, respectively. The delay is also shown to be continuously adjustable from $\Delta t=0$ to $\Delta t=1 /\left(2 f_{m}\right)$ by changing the bias voltage $V_{\mathrm{b}}$, hence the static retardance of the EOM, in agreement with Eq. (9). Different traces are selected and depicted in Fig. 3. By comparing Figs. 3(a)-3(c), a striking observation here is that the pulse pattern remains the same, regardless of the value of $n$ over three orders of magnitude, in agreement with the model. Of course the repetition rate scales as $n$, but it appears that the pulse duration $\tau_{p}$ scales as $1 / n$, leading to a constant duty cycle $\tau_{p} f_{m}$. This is because the harmonics content does not depend on the modulation frequency. Indeed, when $V_{b}$ and $\mathrm{P}_{\mathrm{dc}}$ are kept constant, from $n=1$ to $n=100$ the pulse width decreases from 13 ns down to $130 \mathrm{ps}$ (see Figs. 3(a)$3(\mathrm{c})$ ), yielding a constant $\tau_{p} f_{m}=0.087$. At higher values of $n$ the measurement of the pulse width is limited by the 28 ps-rise time of the detection (see Fig. 3(d) when $f_{m}=3.369 \mathrm{GHz}$ ).

We measure the optical output spectrum by using a 0.06 pm-resolution OSA. As expected, the output optical spectrum contains a dual side-band RF comb, with the seed wavelength at the center and $f_{m}$-harmonics on both sides. In our experimental conditions, we could generate a few dozen harmonics. For example, when $f_{m}$ is $673.7 \mathrm{MHz}$, the total width of the opticalcarried RF comb is $39 \times f_{m}=26.3 \mathrm{GHz}$, as shown in Fig. 4 (a). Raising the gain to higher values leads to parasitic oscillations. When $f_{m}=3.369 \mathrm{GHz}$, Fig. 4(b) shows a $40.4 \mathrm{GHz}$-wide optical frequency comb. In this case, the optical bandwidth is not limited directly by the gain, 
but by the optical filter edges. Moreover, we could observe that changes in the bias voltage influences the harmonics intensities. Namely, at $V_{b}=4 \mathrm{~V}$ when the repetition rate is doubled, odd harmonics are lower than even harmonics. Precisely measuring the short picosecond pulse width (e.g. with an autocorrelator) would permit further analysis of the spectraltemporal relationship.
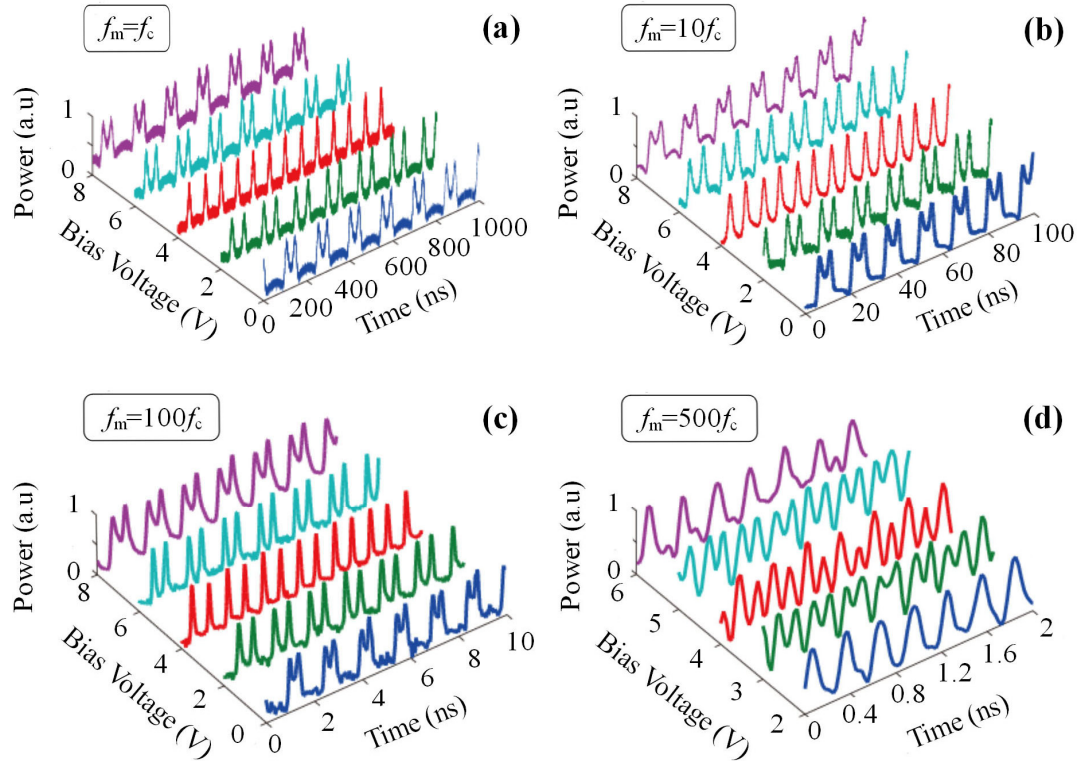

Fig. 3. Experimental pulse doublet regime when $f_{m}=n f_{c}$ : influence of the modulation frequency $f_{m}$ and the bias voltage $V_{b}$. $\mathrm{P}_{\mathrm{dc}}=25 \mathrm{dBm}$; (a) $n=1$; (b) $n=10$; (c) $n=100$; (d) $n=$ 500. In (a)-(b)-(c), $V_{b}=0 \mathrm{~V}$ (blue lines), $2 \mathrm{~V}$ (green lines), $4 \mathrm{~V}$ (red lines), $6 \mathrm{~V}$ (light blue lines), $8 \mathrm{~V}$ (purple lines). In (d), $V_{b}=2 \mathrm{~V}$ (blue line), $3 \mathrm{~V}$ (green line), $4 \mathrm{~V}$ (red line), $5 \mathrm{~V}$ (light blue line), $6 \mathrm{~V}$ (purple line). Pulse FWHM $\tau_{p}$ measured when $V_{b}=4 \mathrm{~V}$ : (a) $\tau_{p}=13 \mathrm{~ns}$, (b) 1.3 ns, (c) $130 \mathrm{ps,} \mathrm{(d)} 80 \mathrm{ps}$ (detection limit).
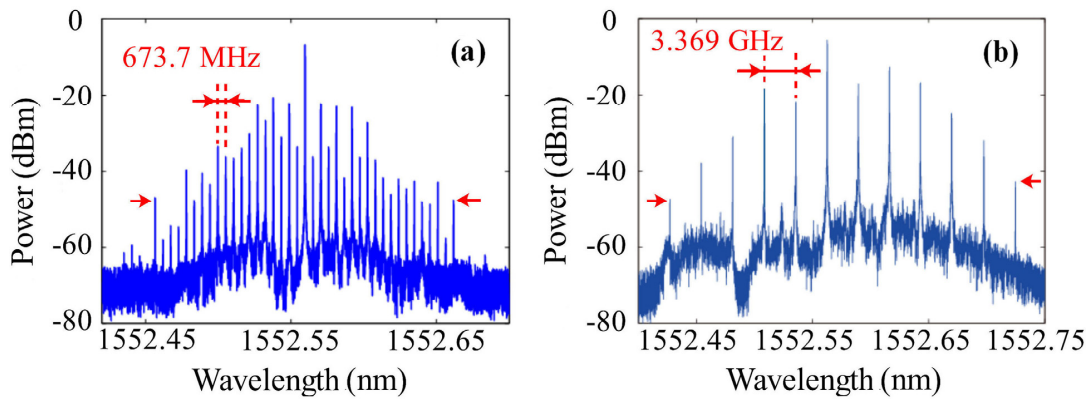

Fig. 4. Optical spectrum of the dual side-band FS loop. (a) $f_{\mathrm{m}}=673.7 \mathrm{MHz}$, the two arrows show the $\pm 19^{\text {th }}$ harmonics. The total comb width is $39 \times f_{\mathrm{m}}=26.3 \mathrm{GHz}$. (b). $f_{\mathrm{m}}=3.369 \mathrm{GHz}$ and the two arrows show the $-5^{\text {th }}$ to $+6^{\text {th }}$ harmonics. The total comb width is $12 \times f_{\mathrm{m}}=40.4$ $\mathrm{GHz}$.

The RF power applied to the EOM is another important factor influencing the doublepulse properties. We first keep $f_{m}=f_{c}=6.737 \mathrm{MHz}$ and $V_{\mathrm{b}}=2 \mathrm{~V}$; Figs. 5(a)-5(c) show that by decreasing $\mathrm{P}_{\mathrm{dc}}$, which corresponds to decreasing the modulation depth $\Gamma_{m}$, we find that (i) the pulse width significantly increases and (ii) the delay decreases. The first feature (i) comes from spectral narrowing due to reduction in the harmonics amplitudes, leading to pulse widening. The second feature (ii) can be understood from the sketch of Fig. 2(b) and Eq. (9): the delay depends on $\Gamma_{\mathrm{m}}$, except for the particular point $\Gamma=\pi / 2$ where $\Delta t=1 /\left(2 f_{m}\right)$ whatever the value of $\Gamma_{m}$. This is confirmed by repeating the experiment with $V_{\mathrm{b}}=4 \mathrm{~V}$ : Figs. 5(d)-5(f) 
show an increase in the pulse width while the delay remains constant, in agreement with Eq. (9).
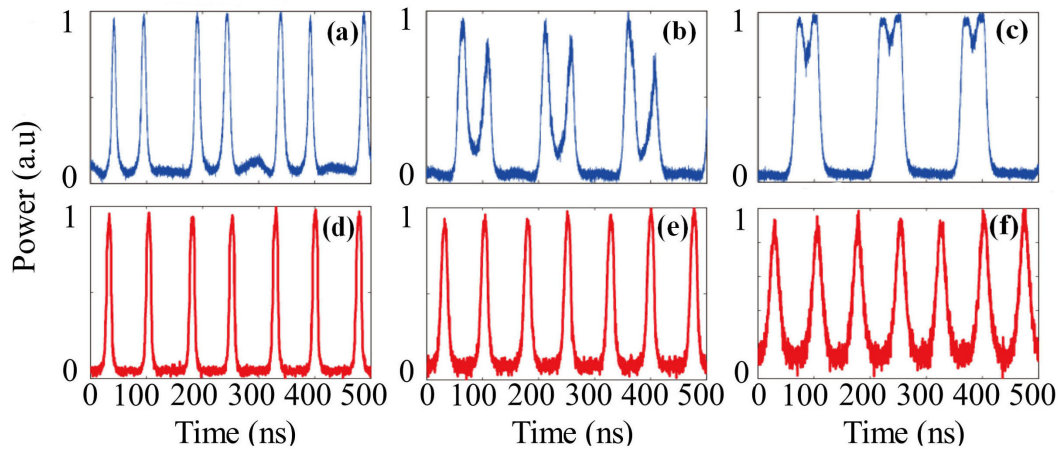

Fig. 5. Influence of RF power on the pulse train. (a),(d) $\mathrm{P}_{\mathrm{dc}}=25 \mathrm{dBm}$; (b),(e) $23 \mathrm{dBm}$ and (c), (f) $21 \mathrm{dBm}$. Upper row (blue curves): $V_{\mathrm{b}}=2 \mathrm{~V}$; lower row (red curves): $V_{\mathrm{b}}=4 \mathrm{~V}$.

\subsection{Rectangle and triangle waveform generation}

Following the preceding conclusions, we find a simple means to generate a rectangle waveform with an adjustable duty cycle. By precisely adjusting $V_{b}$ and $\mathrm{P}_{\mathrm{dc}}$ to make the falling-edge of the first pulse coincides with the rising-edge of the second, we can obtain a rectangle waveform with different duty cycles. As a typical example, we set $f_{m}=1.0039 \mathrm{GHz}$ $\left(149 f_{c}\right.$ ), and adjust $V_{b}$ (resp. $\left.\Gamma\right)$ and $\mathrm{P}_{\mathrm{dc}}\left(\right.$ resp. $\left.\Gamma_{m}\right)$ in the experiments (resp. in the simulation). Figure 6 reports the rectangle waveforms. The FS loop with $\mathrm{P}_{\mathrm{dc}}=21 \mathrm{dBm}$ generates a doublepulse pattern with $\tau_{p} \sim 300 \mathrm{ps}$. We first choose the bias to $V_{b}=1.5 \mathrm{~V}$ in order to transform the double-pulse into a rectangle waveform with a duty cycle of 1:3 (see Fig. 6(a)). When $V_{\mathrm{b}}$ increases to $2.6 \mathrm{~V}$ and $\mathrm{P}_{\mathrm{dc}}$ decreases to $20 \mathrm{dBm}$ simultaneously, we obtain the rectangle waveform with a duty cycle of $1: 2$, i.e. a square wave, as shown in Fig. 6(b). As a last example, Fig. 6(c) shows a rectangle waveform with a duty cycle of 2:3 in the case of $V_{b}=$ $3.3 \mathrm{~V}$ and $\mathrm{P}_{\mathrm{dc}}=19 \mathrm{dBm}$. Corresponding simulations are depicted in Figs. 6(d)-6(f) showing a good agreement with the experimental results.

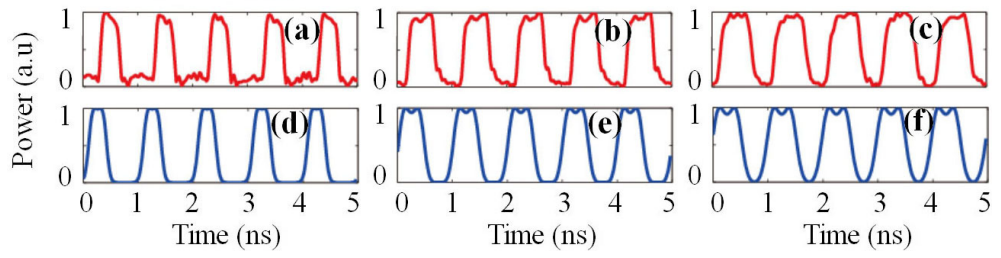

Fig. 6. Rectangle waveform generation; influence of the bias voltage and the RF power on the duty cycle. (a)-(c): Experiment results with (a) $V_{b}=1.5 \mathrm{~V}, \mathrm{P}_{\mathrm{dc}}=21 \mathrm{dBm}$; (b) $V_{b}=2.6 \mathrm{~V}, \mathrm{P}_{\mathrm{dc}}=$ $20 \mathrm{dBm}$ and (c) $V_{b}=3.3 \mathrm{~V}, \mathrm{P}_{\mathrm{dc}}=19 \mathrm{dBm}$. (d)-(f): Simulation results with (d) $\Gamma=1 \mathrm{rad}, \Gamma_{m}=$ 0.6 ; (e) $\Gamma=1.2 \mathrm{rad}, \Gamma_{m}=0.5$ and (f) $\Gamma=1.4 \mathrm{rad}, \Gamma_{m}=0.3$.

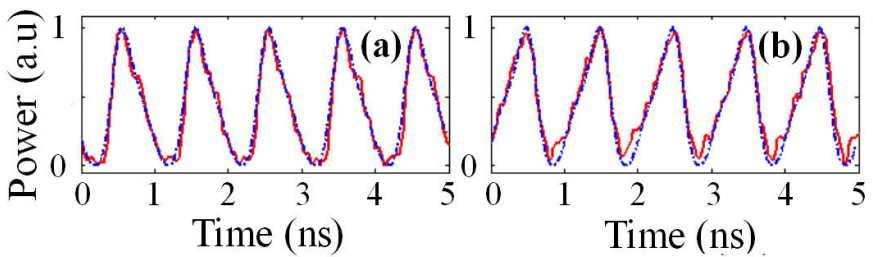

Fig. 7. Saw-tooth waveforms with (a) $f_{\mathrm{m}}=1.0028 \mathrm{GHz}$, (b) $f_{\mathrm{m}}=0.99835 \mathrm{GHz}$. (red line: experiment; dotted blue line: simulation). 
The waveform generation is not limited to the rectangle case. It is well known that AOFS loops output waveform relies on the shifting frequency $f_{\mathrm{m}}$, and RF power, and the loop length. Here, we find saw-tooth waveforms when we slightly detune the modulation frequency of the EOM off an integer value $n f_{\mathrm{c}}$. For example, we set $f_{\mathrm{m}}=1.0028 \mathrm{GHz}$ and $0.99835 \mathrm{GHz}$, respectively, and find the triangle waveforms reproduced in Figs. 7(a)-7(b). In the experiment, we keep $V_{\mathrm{b}}=3 \mathrm{~V}$ and $\mathrm{P}_{\mathrm{dc}}=25 \mathrm{dBm}$. A good agreement with the experiment is obtained when $\Gamma_{m}=0.6$ and $\Gamma=1.2$ in the simulations (see blue dotted lines in Fig. 7), confirming the validity of the linear model.

\section{Mode-locked pulse doublets generation from the un-seeded loop}

For the sake of completeness, we investigate shortly the loop behavior without the seed laser when the gain exceeds the losses, i.e., when the loop is driven above laser threshold. It has long been shown that phase or loss modulation of lasers lead to the mode-locked regime when the modulation frequency is a multiple of the cavity free-spectral range [20,21]. In particular picosecond pulse generation was demonstrated in erbium-doped fiber lasers using phase modulators [22-24]. However, contrary to phase-modulated mode-locked lasers, here it appears that amplitude modulation leads to picosecond pulse doublet operation, a situation that, to the best of our knowledge, has never been reported. The EDFA in our set-up allows us to raise the gain up to the laser threshold. We show at first the experimental results when $f_{\mathrm{m}}=$ $f_{\mathrm{c}}$ in Fig. 8 (in this case the fiber loop was slightly reduced, leading to $f_{\mathrm{c}}=7.611 \mathrm{MHz}$ ). Obviously, the double-pulse regime still exists in this un-seeded mode-locked operation. Furthermore, it is interesting to note that the delay time is continuously tunable and also obeys Eq. (9), as shown for example in Fig. 8 with two different values of the bias voltage. At variance with the seeded case of Section 3, in which the pulse width is about $13 \mathrm{~ns}$, here the laser pulses can be reduced down to $\tau_{p}=425$ ps (when $V_{\mathrm{b}}=4 \mathrm{~V}$ ). Also in agreement with the linear model, the un-seeded FS ring laser generates sub-nanosecond pulses when $f_{\mathrm{m}}=n f_{\mathrm{c}}$ with $n \neq 1$ (harmonic mode-locking). Figure 9 evidences this double-pulse regime, without seed laser, up to $n=100$, where harmonic mode-locked pulses have a $70 \mathrm{ps}$ duration.
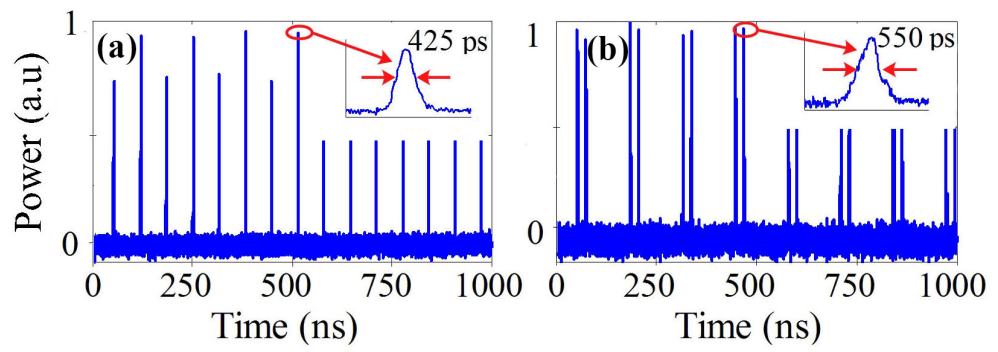

Fig. 8. Resonant mode-locked double-pulse operation with $f_{\mathrm{m}}=7.611 \mathrm{MHz}$. (a) $V_{\mathrm{b}}=4 \mathrm{~V}$. (b) $V_{\mathrm{b}}$ $=0 \mathrm{~V}$.
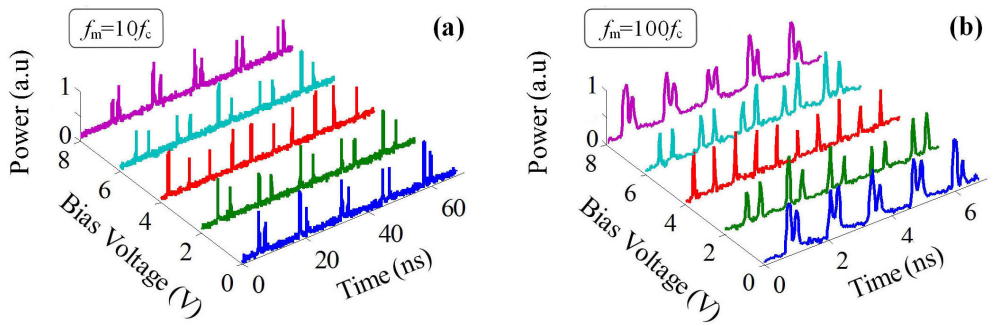

Fig. 9. Harmonic mode-locked double-pulse operation. Experimental laser output when (a) $f_{\mathrm{m}}$ $=76.11 \mathrm{MHz}, \tau_{p}=210 \mathrm{ps}$ (b) $f_{\mathrm{m}}=761.1 \mathrm{MHz}, \tau_{p}=70 \mathrm{ps}$. 


\section{Conclusion}

To sum up, a FSL containing an amplitude modulator and an amplifier produces pulses in the sub-nanosecond range when seeded by a cw laser, if the loop length is a multiple of the RF modulation wavelength applied to the modulator. This experiment shows an alternative approach to AOFS loops, taking advantage of the inherent bandwidth and tunability of the EOM. We have evidenced analytically and experimentally an original regime of pulse doublets that finds its origin in the transfer function of the EOM. It is found that the delay between the pulses is simply governed by the modulator's bias. In addition, by properly setting the modulation frequency, adjustable rectangle and saw-tooth waveforms can be obtained. Finally the double-pulse regime survives to above-threshold operation: without the seed, mode-locked picosecond pulse doublets are also generated with an adjustable delay.

Beyond this first demonstration, careful characterization and reduction of jitter issues need further work that is under progress. Designing a loop with all polarization-maintaining fiber components, as well as acoustic and thermal isolation, would improve the stability.

The proof-of-concept demonstrated here could be extended to integrated photonics since optical rings, filters, and EOMs can be integrated on photonic platforms. This EOM-based FS loop could address applications already envisioned with FS loops, like high-repetition rate pulse trains [11], RF waveform generation [13,25] and processing [14], or ranging [18,26,27]. Besides, such a scheme may be considered when designing pump-probe experiments, since the delay can be voltage-controlled without using moving delay lines.

\section{Funding}

Chinese Scholarship Council (CSC), Région Bretagne, FEDER (EU), and Rennes Metropole, in the framework of CPER SOPHIE-Photonique.

\section{Acknowledgments}

The authors thank H. Guillet de Chatellus for stimulating discussions.

\section{References}

1. J. P. Yao, "Microwave photonics," J. Lightwave Technol. 27(3), 314-335 (2009).

2. J. Capmany and D. Novak, "Microwave photonics combines two worlds," Nat. Photonics 1(6), 319-330 (2007).

3. J. Capmany, J. Mora, I. Gasulla, J. Sancho, J. Lloret, and S. Sales, “Microwave photonic signal processing," J. Lightwave Technol. 31(4), 571-586 (2013).

4. C. H. Lee, Microwave Photonics, $2^{\text {nd }}$ ed. (CRC, 2013).

5. X. S. Yao and L. Maleki, "Optoelectronic oscillator for photonic systems," IEEE J. Quantum Electron. 32(7), 1141-1149 (1996).

6. J.-W. Shi, C.-B. Huang, and C.-L. Pan, "Millimeter-wave photonic wireless links for very high data rate communication," NPG Asia Mater. 3(4), 41-48 (2011).

7. H.-P. Chuang and C.-B. Huang, "Generation and delivery of 1-ps optical pulses with ultrahigh repetition-rates over 25-km single mode fiber by a spectral line-by-line pulse shaper," Opt. Express 18(23), 24003-24011 (2010).

8. J. Wells, "Faster than fiber: the future of multi-Gb/s wireless," IEEE Microw. Mag. 10(3), 104-112 (2009).

9. F. V. Kowalski, S. J. Shattil, and P. D. Hale, "Optical pulse generation with a frequency shifted feedback laser," Appl. Phys. Lett. 53(9), 734-736 (1988).

10. H. Sabert and E. Brinkmeyer, "Pulse generation in fiber lasers with frequency shifted feedback," J. Lightwave Technol. 12(8), 1360-1368 (1994).

11. H. Guillet de Chatellus, O. Jacquin, O. Hugon, W. Glastre, E. Lacot, and J. Marklof, "Generation of ultrahigh and tunable repetition rates in CW injection-seeded frequency-shifted feedback lasers," Opt. Express 21(13), 15065-15074 (2013).

12. H. Guillet de Chatellus, E. Lacot, W. Glastre, O. Jacquin, and O. Hugon, "Theory of Talbot lasers," Phys. Rev. A 88(3), 033828 (2013).

13. A. Kanno, I. Morohashi, T. Kuri, I. Hosako, T. Kawanishi, Y. Yasumura, Y. Yoshida, and K. Kitayama, "16Gbaud QPSK Radio Transmission using Optical Frequency Comb with Recirculating Frequency Shifter for 300GHz RoF Signal," in IEEE International Topical Meeting on Microwave Photonics, 2012, 298-301.

14. F. Tian, X. Zhang, J. Li, and L. Xi, "Generation of 50 stable frequency-locked optical carriers for tb/s multicarrier optical transmission using a recirculating frequency shifter," J. Lightwave Technol. 29(8), 10851091 (2011). 
15. H. Guillet de Chatellus, L. R. Cortés, and J. Azaña, "Optical real-time Fourier transformation with kilohertz resolutions," Optica 3(1), 1-8 (2016).

16. C. Schnébelin and H. Guillet de Chatellus, "Optical spectral shaping with MHz resolution for arbitrary RF waveform generation," in Conference on Lasers and Electro-Optics, OSA Technical Digest (online) (Optical Society of America, 2018), paper SM1B.7.

17. L. Wang and S. LaRochelle, "Talbot Laser with Tunable GHz Repetition Rate using an Electro-Optic Frequency Shifter," in Conference on Lasers and Electro-Optics, OSA Technical Digest (online) (Optical Society of America, 2017), paper JW2A.66.

18. H. Yang, M. Brunel, H. Zhang, M. Vallet, C. Zhao, and S. Yang, "RF up-conversion and waveform generation using a frequency-shifting amplifying fiber loop, application to Doppler velocimetry," IEEE Photonics J. 9(6), 7106609 (2017).

19. A. Yariv, "Universal relations for coupling of optical power between microresonators and dielectric waveguides," Electron. Lett. 36(4), 321-322 (2000).

20. J. Hirano and T. Kimura, "Multiple mode-locking of lasers," IEEE J. Quantum Electron. 5(5), 219-225 (1969).

21. A. E. Siegman, Lasers (Mill Valley, 1986).

22. J. D. Kafka, T. Baer, and D. W. Hall, "Mode-locked erbium-doped fiber laser with soliton pulse shaping," Opt. Lett. 14(22), 1269-1271 (1989).

23. A. Takada and H. Miyazawa, " $30 \mathrm{GHz}$ picosecond pulse generation from actively mode-locked erbium-doped fibre laser," Electron. Lett. 26(3), 216-217 (1990).

24. D. D. Hudson, K. W. Holman, R. J. Jones, S. T. Cundiff, J. Ye, and D. J. Jones, "Mode-locked fiber laser frequency-controlled with an intracavity electro-optic modulator," Opt. Lett. 30(21), 2948-2950 (2005).

25. H. Guillet de Chatellus, L. Romero Cortés, C. Schnébelin, M. Burla, and J. Azaña, "Reconfigurable photonic generation of broadband chirped waveforms using a single CW laser and low-frequency electronics," Nat. Commun. 9(1), 2438 (2018).

26. H. Zhang, M. Brunel, M. Romanelli, and M. Vallet, "Green pulsed lidar-radar emitter based on a multipass frequency-shifting external cavity," Appl. Opt. 55(10), 2467-2473 (2016).

27. J. Clement, C. Schnébelin, H. G. de Chatellus, and C. R. Fernández-Pousa, "Laser ranging using coherent pulse compression with frequency shifting loops," Opt. Express 27(9), 12000-12010 (2019). 